\title{
Malignant Pericardial Effusion
}

National Cancer Institute

\section{Source}

National Cancer Institute. Malignant Pericardial Effusion. NCI Thesaurus. Code C7717.

Presence of fluid in the pericardial space as a complication of malignant disease. 